\title{
Rheological properties of inverse emulsions stabilized by ethanolamides of tall oil fatty acids
}

\author{
V. A. Yanovsky ${ }^{1, *}$, M. O. Andropov ${ }^{1,2}$, R. S. Fakhrislamova ${ }^{1,3}$, R. A. Churkin ${ }^{1,3}$, \\ K. M. Minaev ${ }^{3}$ and O. S. Ulyanova ${ }^{3}$ \\ ${ }^{1}$ Siberian Physical-Technical Institute of Tomsk State University, 1 Novosobornaya sq., Tomsk, \\ 634050, Russia \\ ${ }^{2}$ Seversk Technological Institute of National Research Nuclear University "MEPhI", \\ 65 Kommunisticheski avenue, Seversk, 636036, Russia \\ ${ }^{3}$ National Research Tomsk Polytechnic University, 30 Lenin Ave., Tomsk, 634050, Russia
}

\begin{abstract}
The paper presents the comparative analysis of inverse emulsion rheological behavior stabilized by monoethanolamide and diethanolamide of tall oil fatty acids, which are promising reagents for petroleum engineering. Flow curves of emulsions and volume ratio of dispersed phase have been obtained. The analysis of the dependence of Herschel-Bulkley model parameters on concentration of emulsifiers has been carried out. It is proved that emulsifiers affect weakly on the rheological parameters in high water phase content emulsions, while in cut-back emulsions their impact is registered to be stronger. It has been found that rheological parameters of the emulsions stabilized by monoethanolamide throughout the range of concentration are considerably higher than the samples on the base of diethanolamide. The experimental data proved the key impact of viscosity of dispersion medium on rheological behavior of cut-back emulsions
\end{abstract}

\section{Introduction}

Inverse emulsions also known as invert emulsions or water-in-oil emulsions are widely used in various branches of industry, for example, food, cosmetic and pharmacy, as well as building and chemistry [1]. Also they are used for petroleum engineering [2-4]. Oil-base muds (OBM) or the inverse emulsions of the mineralized water solutions in hydrocarbon phase due to their unique technological parameters are very essential for drilling in complex environments, producing formations completion and as well-killing fluids.

In recent decades inverse emulsions are becoming increasingly important to enhance oil recovery [5-7]. Their efficiency is defined by their nature: they are viscous water-proofing fluids, which firstly, enhance swabbing needed for an oil drive, and secondly, get remaining oil in the pipes. In addition, in high-permeability watered layer water is sucked by the emulsion, which increases its viscosity, puts watertight barrier and as a consequence, decreases the water relative permeability. Such parameter as viscosity of inverse emulsions

* Corresponding author: mailto:yavatpu@yandex.ru 
can be easily adjusted in various ways, the simplest of which is to change the ratio of the water phase to hydrocarbon phase. Furthermore, the viscosity of such emulsions can be controlled the type of emulsifier, its concentration, and addictives, stabilized the emulsions and structured within a dispersion medium. Inverse emulsion provides additional $5-20$ tons of oil per 1 ton of drilling mud [8].

Rheological parameters of inverse emulsions define their efficiency, when they are used as drilling muds, for example, OBM or to enhance an oil drive in various canals (wellbore, pores and reservoir-scale fractures) as well as in the process of cuttings.

In previous works $[9,10]$ the authors carried out the synthesis of such emulsifiers as ethanolamides of tall oil fatty acid, and the stability of these emulsions was studied. This paper is a continuation of these studies and its purpose is to study of rheological parameters of inverse emulsions stabilized by ethanolamides of tall oil fatty acids.

\section{Methods and materials}

\subsection{Reagents and materials}

Monoethanolamide (MATO) and Diethanolamide (DATO) of tall oil fatty acid were produced by direct amidation of distilled tall oil (Monoethanolamide and Diethanolamine, respectively) at $150-160{ }^{\circ} \mathrm{C}$ under vacuum $(60-70 \mathrm{~mm} \mathrm{Hg})$. The synthesis was carried out to full dewatering of the reaction mixtures (approximately 3-4 hours) for MATO $2.5 \mathrm{mg} \mathrm{KOH} / \mathrm{g}$, DATO $-4.0 \mathrm{mg} \mathrm{KOH} / \mathrm{g}$. The following materials are used such as the distilled tall oil, produced by OAO "Selenginskij cellyulozno-kartonnyj kombinat", diesel fuel (DT-EVRO), class 3, type I-III (winter) according to GOST R 52368-2005 with $2.13 \mathrm{~mm}^{2} / \mathrm{s}$ kinematic viscosity (GOST 33-2000) at $40{ }^{\circ} \mathrm{C}$, and such p.a. reagents as chloride of calcium anhydrous $\left(\mathrm{CaCl}_{2}\right)$ and chloride of sodium $(\mathrm{NaCl})$.

\subsection{Preparation of emulsions}

Hydrocarbon phase was preliminary prepared by estimated amount of emulsifier solution in diesel fuel, while water phase was prepared by solution of $\mathrm{CaCl}_{2}$ and $\mathrm{NaCl}$ into a delivery flask. The emulsions were prepared by mechanical dispersion of water phase in hydrocarbon phase by Triple-Spindle Drink Mixer HAMILTON BEACH HMD 400 with $14000 \mathrm{rpm}$ at room temperature. Estimated amount of emulsifier in diesel fuel was put into a cup of the Drink Mixer. Then it was mixed with addition of some portions of required amount of water phase with their full emulsionizing. When the last portion of water phase was added, the emulsions continued to be mixed for $5 \mathrm{~min}$. Then the emulsions were put under vacuum in a vacuum rotary evaporator at room temperature for about $20 \mathrm{~min}$ to full air elimination.

\subsection{Rheological measurements}

Rheological measurements of emulsion were made immediately after preparation and degassing by viscosimeter Brookfield DV3T LV with Brookfield Thermosel ${ }^{\circledR}$ System at speed range of $10-250 \mathrm{rpm}$. Before measuring each sample was thermostated at specified temperature for $30 \mathrm{~min}$. The accuracy of temperature control is $\pm 0.2{ }^{\circ} \mathrm{C}$. Brookfield Rheocalc T 1.1.13 Software is used for data reduction process.

Kinematic viscosity of emulsifier solutions was registered according to ASTM D445 with accuracy $\pm 1.0 \%$ by Ubbelohde viscometer where a capillary diameter is $0.56 \mathrm{~mm}$. 


\subsection{Microscopy}

Microphotography of the emulsions were taken by Nikon unveils Coolscope II microscope.

\section{Results and discussion}

The authors have investigated the rheological parameters of inverse emulsions, prepared by using two nonionic emulsifiers of alkanolamide class: Monoethanolamide (MATO) and Diethanolamide (DATO) of tall oil fatty acids. Emulsifier range of concentrations, volume ratio and contain of the dispersed phase were chosen in compliance with real emulsions, used as drilling muds in petroleum engineering. Emulsifier concentration $(c)$ was varied in the range of $0.75-15 \%$ (wt) to hydrocarbon phase or $0.192-6.764 \%$ (wt) to afforded emulsion. The high-concentrated emulsions with volume ratio of dispersed phase $(\varphi)$ corresponded to $0.7,0.6$ and 0.5 were studied, where the last was in the form of the mineralized water solution with $32 \mathrm{~g} / \mathrm{dm}^{3}$ of $\mathrm{CaCl}_{2}$ and $16 \mathrm{~g} / \mathrm{dm}^{3} \mathrm{NaCl}$.

The Herschel-Bulkley model was used to describe the rheological behavior of the inverse emulsions. This model demonstrates well the rheological parameters of nonNewtonian fluids and is often used to analyze high-concentrated emulsion behavior [11, 12]. The Herschel-Bulkley model is commonly written as

$$
\tau=\tau_{\mathrm{T}}+K \cdot \dot{\gamma}^{n},
$$

where $\tau$ is the shear stress, $\mathrm{Pa} ; \dot{\gamma}$ the shear rate, $\mathrm{s}^{-1} ; \tau_{\mathrm{T}}$ the yield stress, $\mathrm{Pa} ; K$ the consistency index, and $n$ the flow index.

The flow curves of inverse emulsions at $25^{\circ} \mathrm{C}$ are shown in figure 1 with different volume ratios $(\varphi)$ of dispersed phase and different emulsifier concentration (c).

In $70 \%$ water phase emulsions (fig.1a), the flow curves of the studied emulsifiers are arranged in pairs, depending on their concentration. When $(c)$ index is high, DATO provides more viscous emulsion than MATO. When (c) index is lower than 5\%, inversion of behavior is observed: MATO provides more viscous emulsions. In general, the impact of chemical nature of emulsifiers on rheological behavior of high-concentrated emulsion can be considered negligible. However, the situation radically changes with solution of the emulsion by dispersed medium (fig. $1 \mathrm{~b}$ and 1c). The samples stabilized by MATO, demonstrate much higher viscosity than the samples with DATO. In addition, the higher emulsifier concentration is, the more differences in viscosity are. The viscosity of the samples on the base of MATO is higher than in the entire range of emulsifier concentration and shear rate. For example, according to Figure $1 \mathrm{~b}$, the emulsion with $10 \%$ of MATO has practically the same rheological parameters as the emulsion with $15 \%$ of DATO.

Then the dependence of the Herschel-Bulkley model parameters on emulsifier concentration in the emulsions was analyzed. The registered data are shown in Figure 2.

The dependences of the yield stress $\left(\tau_{\mathrm{T}}\right)$ on emulsifier concentration (c) (fig. 2a) are predictably considered to have a form of rising curves. More intense ascend of $\tau_{\mathrm{T}}$ index is expectedly observed in higher water phase emulsions, which can be explained by increasing contacts between droplets at increasing dispersed phase content [11]. It was registered the weak impact of chemical nature of emulsifiers on the yield stress for high-concentrated emulsions $(\varphi=0.7)$. MATO provided higher $\tau_{\mathrm{T}}$ index in cut-back emulsions than DATO, and the difference increased with increasing $c$ index. Therefore, the dependence $\tau_{\mathrm{T}}-c$ with the same $\varphi$ are considered to be divergent curves for each pair of emulsifiers.

The dependence of the consistency index curves $(K)$ on emulsifier concentration $(c)$ index shown in Figure 2b is similar to curves $\tau_{\mathrm{T}}-c$ mentioned-above. The one difference is the higher $K$ index for DATO, observed in high-concentrated emulsions. This fact naturally follows from the flow curves of the emulsions, shown in Figure 1a. 


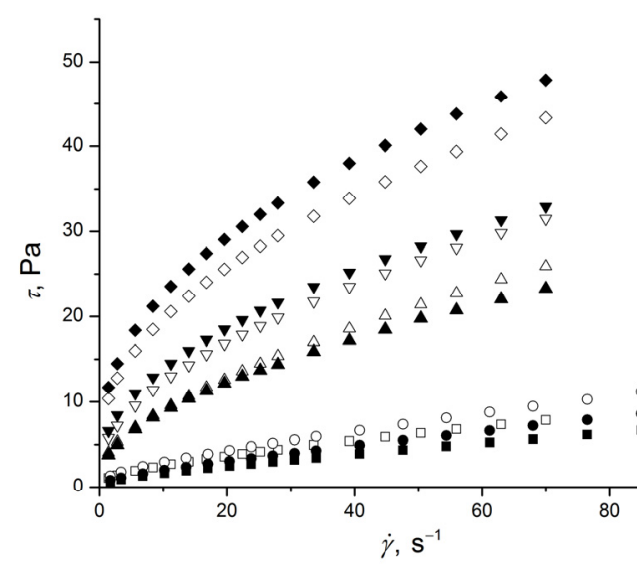

a)

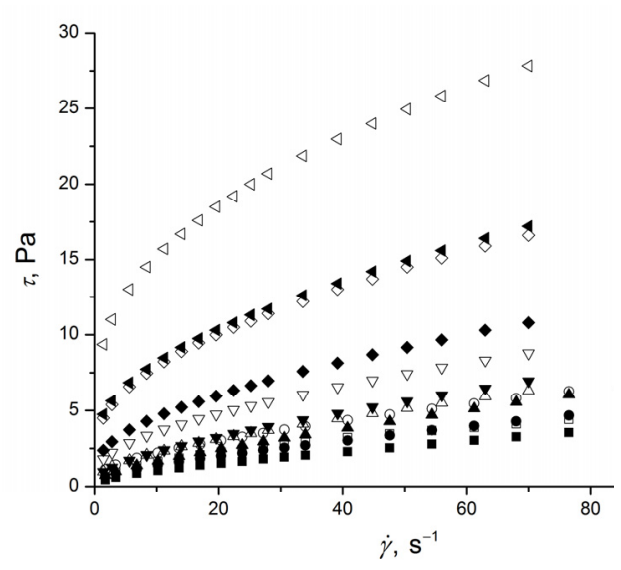

b)

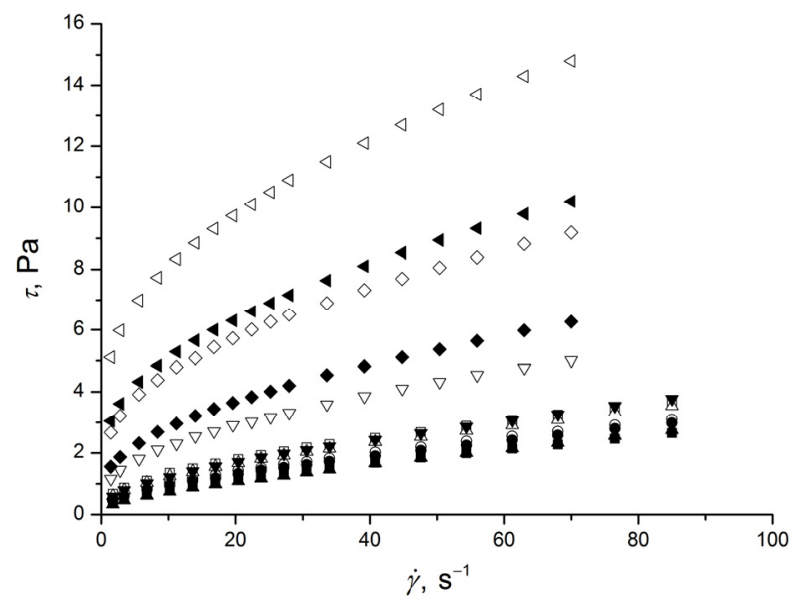

c)

Fig. 1. The flow curves of inverse emulsions $\left(25^{\circ} \mathrm{C}\right)$ : a) $\varphi=0.7$; b) $\varphi=0.6$; c) $\varphi=0.5$ ( $\square-$ MATO; - DATO; $\square-0.75 \%$; ○ $-1 \% ; \Delta-2 \%$; $\nabla-5 \% ; \diamond-10 \% ; \triangleleft-15 \%$; the emulsifier concentration in hydrocarbon phase is shown)

A special feature of flow index dependence on emulsifier concentration (fig. 2c) is maximum value at low $c$ index (not more than $1 \%$ in the emulsion). And increasing of water phase in emulsion gives maximum values at lower $c$ index. Further behavior of the curves also depends on $\varphi$ : with an increase of $c$ index the flow index sharply decreases from $\approx 0.8$ at $c=0.257$ to $0.55-0.65$ at $c=0.514$ in high-concentrated emulsions. In cut-back emulsions a decrease of $n$ index from maximum point is smooth. With further increasing $c$ index the curves of all emulsions reaches some maximum $n$ index and then, $c$ index becomes independent from the flow index $(n)$.

It should be noted that under equal conditions DATO provides maximum flow index in more concentrated emulsions, while MATO - in less concentrated emulsions. 


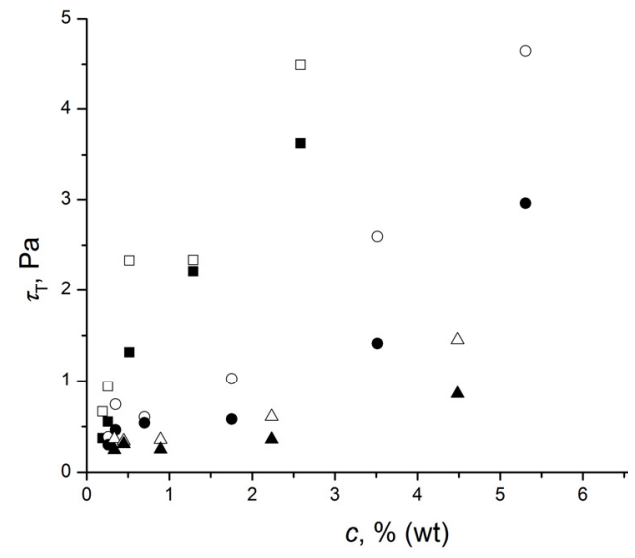

a)

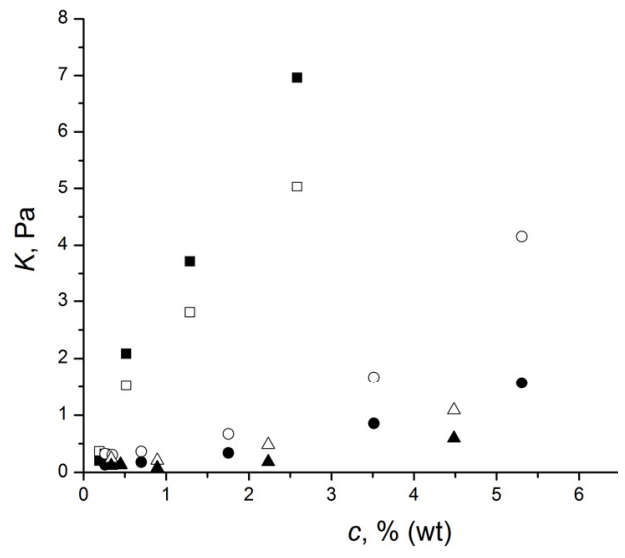

b)

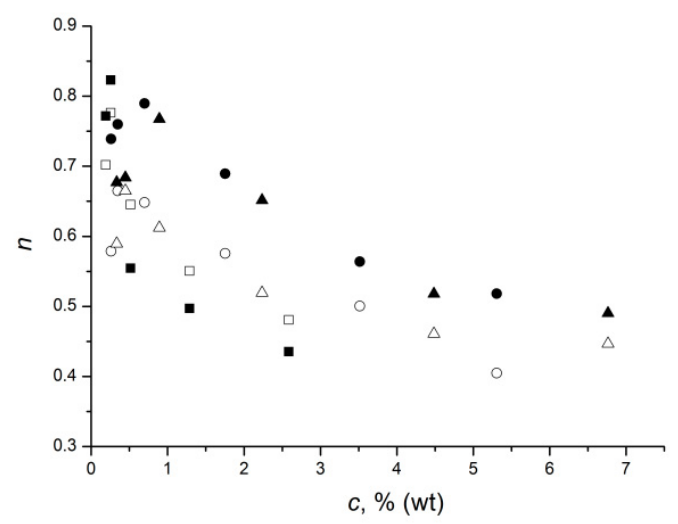

c)

Fig. 2. The dependence of rheological parameters on the emulsifier concentration at $25^{\circ} \mathrm{C}$ a) the yield stress $\left(\tau_{\mathrm{T}}\right)$; b) the consistency index $(K)$; c) the flow index $(n)$ ( $\square-\mathrm{MATO}$; - DATO; $\square-\varphi=0.7 ; \circ-\varphi=0.6 ; \Delta-\varphi=0.5$; the emulsifier concentration in emulsion is shown)

When analyzing the rheological behavior of the emulsions, it is necessary to remember about the simultaneous impact of complex factors. For example, Sherman [13] defined approximately two dozens of these factors. Concerning studied emulsions it is possible to distinguish the following factors: the average particle diameter, polydispersity index, continuous-phase viscosity, and droplet flocculation, as well as surface tension, thickness and rheological parameters of adsorption layer.

A microstructure of the emulsions was studied to evaluate the impact of droplets dimension and their polydispersity index. To compare these parameters, the samples of the emulsions, stabilized by MATO and DATO in the same concentration (при $\varphi=0.6$ ) which had maximum differences in rheological behavior were selected (fig. 1b). 


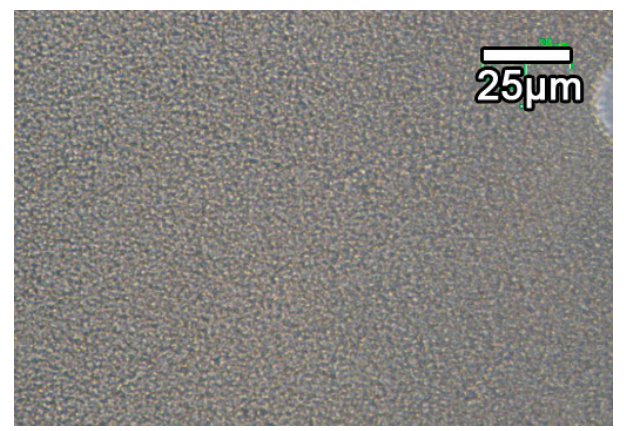

a)

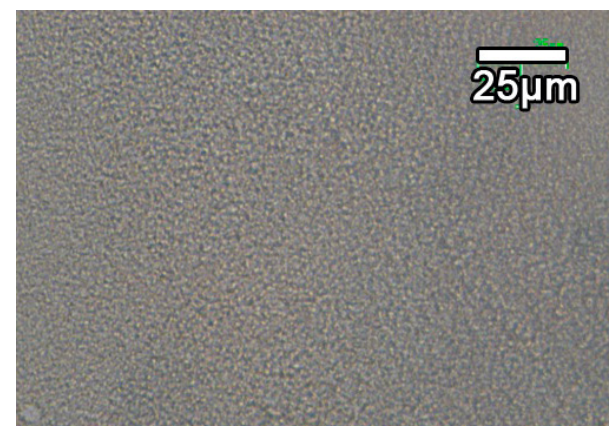

b)

Fig. 3. The microphotographs of emulsions at $c=10 \%$ (hydrocarbon phase), $\varphi=0.6$ : a) MATO; b) DATO

According to microphotographs shown in Figure 3 there are no significant differences in microstructure of studied emulsions. Therefore, the factor of particle diameter, in our opinion, is not considered to be important for studied emulsions. The impact of rheological parameters of dispersion phase is assumed to be a more important factor. These assumptions are confirmed by experimental data. Table 1 shows kinematic viscosity index of emulsifier solution in diesel fuel of different concentration. According to Table 1, low concentrated solutions (up to $5 \%$ ) have similar viscosity indexes generally correlate to rheological parameters of corresponding emulsions. With further increasing emulsifier concentration, nonlinear growth of viscosity is observed, and the viscosity of emulsions with MATO increases faster than DATO emulsions. A similar dependence was registered in cut-back emulsions $(\varphi=0.5-0.6)$. It should be noted that this dependence is not observed in high-concentrated emulsions $(\varphi=0.7)$. As mentioned above, at high values of $c$ DATO forms slightly more viscous emulsions than MATO. Consequently, other factors are predominating in high-concentrated emulsions. Taking into account the more density of droplets there, it might be assumed that parameters of adsorption layer of emulsifier at phase boundary is, probably, more important, but this hypothesis needs further confirmation.

Table 1. The kinematic viscosity index of emulsifier solution in diesel fuel at $25^{\circ} \mathrm{C}$.

\begin{tabular}{|c|c|c|}
\hline $\boldsymbol{c}, \boldsymbol{\%}(\mathbf{w t})$ & MATO & DATO \\
\hline 0 & 2.91 & - \\
\hline 0.75 & 2.98 & 3.00 \\
\hline 1 & 3.00 & 3.02 \\
\hline 2 & 3.16 & 3.11 \\
\hline 5 & 3.45 & 3.52 \\
\hline 10 & 4.25 & 4.15 \\
\hline 15 & 5.70 & 5.27 \\
\hline
\end{tabular}

\section{Conclusions}

To sum up, the rheological behavior of inverse emulsions, stabilized by monoethanolamide and diethanolamide of tall oil fatty acids have been analyzed in the paper. The flow curve of different concentrated emulsions and volume ratio of dispersed phase have been described, as well as the dependence of Herschel-Bulkley model parameters on concentration of emulsifiers. It has been proved that chemical nature of emulsifier affects 
weakly on the rheological parameters in high-concentrated emulsions $(\varphi=0.7)$. In such emulsions DATO (more than $5 \%$ content) provides higher viscosity and minimum flow index. In cut-back emulsions the impact of rheological parameters on the structure of the emulsifier varies significantly. The rheological parameters of cut-back emulsions, stabilized by MATO in the entire range of concentration are appreciably higher than parameters of DATO samples. The experimental data have proved the key impact of viscosity of dispersion medium on rheological behavior of cut-back emulsions.

This work was supported by Federal Target Program «Research and development on priority directions of scientific-technological complex of Russia for 2014-2020» (RFMEFI57814X0068).

\section{References}

1. T. F. Tadros, Emulsion Science and Technology (Wiley-VCH Verlag GmbH \& Co. KGaA, Weinheim, 2009)

2. V. N. Glushchenko The inverse emulsion and suspension in the oil and gas industry [Obratnye ehmul'sii $i$ suspenzii v neftegazovoj promyshlennosti] (Intercontact Nauka, Moscow, 2008) (in Russian)

3. V. I. Tokunov, I. B. Heyfets Hydrophobically-emulsion drilling muds [Gidrofobnoehmul'sionnye burovye rastvory] (Nedra, Moscow, 1983) (in Russian)

4. A. Ya. Soloviev, V. A. Dokichev, G. V. Konesev, Neftegazovoe delo. 2, 67 (2004) (in Russian)

5. M. K. Rogachev, D. V. Mardashov, A. R. Mavliev, Neftegazovoe delo. 3, 180 (2011) (in Russian)

6. A. N. Beregovoj, M. I. Amerkhanov, S. G. Rakhimova, E. P. Vasiljev, Neftyanoe khozyaystvo - Oil Industry. 8, 116 (2010)

7. V. A. Kotelnikov, M. F. Romantsev, V. B. Zavolzhskiy, Neftyanoe khozyaystvo - Oil Industry. 6, 110 (2002)

8. I. M. Araslanov, Inzhenernaya praktika. 7, 75 (2011) (in Russian)

9. V. A. Yanovsky, R. A. Churkin, M. O. Andropov, N. I. Kosova, Vestnik Tomskogo gosudarstvennogo universiteta. 370, 194 (2013) (in Russian)

10. K. Minaev, A. Epikhin, D. Novoseltsev, M. Andropov, V. Yanovsky, O. Ulyanova, IOP Conf. Ser.: Earth Environ. Sci. 21, 012033 (2014)

11. S. R. Derkach Rheology of emulsions [Reologiya emulsiy] (Nauka, St. Petersburg, 2012) (in Russian)

12. T. A. Dyakina, S. R. Derkatch, S. M. Levachev, Vestnik Moskovskogo Universiteta. Khimija. vol. 59, 1, 42 (2004) (in Russian)

13. R. V. Petersen Emulsion science, ed Philip Sherman (Academic Press, London, 1968) 